\title{
Clinical Presentation, Management, and Prognosis of Pseudogout in Joint Arthroplasty: A Retrospective Cohort Study
}

\author{
Merit P. George ${ }^{1}$, Floranne C. Ernste², Aaron Tande³, Douglas Osmon³, Tad Mabry4, Elie F. Berbari ${ }^{3 凶}$ \\ 1. Mayo Clinic School of Medicine. 200 1st St SW, Rochester, MN 55905. \\ 2. Division of Rheumatology, Department of Internal Medicine, Mayo Clinic School of Graduate Medical Education. 200 1st St SW, Rochester, MN 55905. \\ 3. Division of Infectious Diseases, Department of Internal Medicine, Mayo Clinic School of Graduate Medical Education. 200 1st St SW, Rochester, MN 55905. \\ 4. Division of Orthopedic Surgery, Department of Surgery, Mayo Clinic School of Graduate Medical Education. 200 1st St SW, Rochester, MN 55905 \\ $\square$ Corresponding author: \\ (1) Ivyspring International Publisher. This is an open access article distributed under the terms of the Creative Commons Attribution (CC BY-NC) license \\ (https://creativecommons.org/licenses/by-nc/4.0/). See http://ivyspring.com/terms for full terms and conditions.
}

Received: 2018.09.16; Accepted: 2018.12.06; Published: 2019.01.01

\begin{abstract}
Introduction: Calcium pyrophosphate deposition disease (CPPD), or pseudogout, is rare in prosthetic joints, but can mimic prosthetic joint infection (PJI) according to case reports. The purpose of this case series is to describe the demographics, presentation, management, and outcomes of a cohort of these patients seen at our academic medical center.

Methods: Patients with post-implant pseudogout, who were evaluated at our medical center between January 1, 2000 and June 30, 2016, were identified from our EHR. Data pertaining to demographics, presentation, management, and outcomes were abstracted, and patients were categorized into two groups based on presence of concomitant infection along with positive CPDD findings in synovial fluid.

Results: 22 patients were included. $90.9 \%$ of cases involved a TKA. The most common indication for arthroplasty was degenerative joint disease. Only four patients had a history of previous gout or pseudogout, three of which belonged to the group with no evidence of concomitant joint infection. Clinical features for patients without concomitant infection included pain (100\%), swelling at the joint (88.9\%), redness (33.3\%), fever (22.2\%), and decreased range of motion (100\%). $45.5 \%$ of patients received antibiotics prior to joint aspiration ( $44.4 \%$ of patients with negative synovial fluid cultures, $46.2 \%$ of patients with concomitant infection).

Conclusion: Our study suggests similar clinical presentation between post-implant pseudogout and PJI. Among patients with pseudogout as well as in those with PJI, the first dose of antibiotics should not be given before sampling for synovial culture. Unfortunately, many patients receive antibiotics prior to culture ascertainment, which raises concern for antibiotic overuse.
\end{abstract}

Key words: pseudogout, prosthetic infection, arthroplasty

\section{Introduction}

Previous studies have estimated that around 4.7 million Americans were living with total knee arthroplasty (TKA) in 2010, with an additional 2.5 million living with total hip arthroplasty (THA) in the same year [1]. Among all living adults between the age of $80-89$, about $6 \%$ had a history of total hip replacement while around $10 \%$ have a history of total knee replacement [1]. Given the notable prevalence rates for these procedures, complications of prosthetic implants have been of clinical importance. Perhaps most severe among these complications are infectious complications of the implanted joints, referred to as prosthetic joint infection (PJI). Data from the Nationwide Inpatient Sample suggests that the annual incidence rate of PJI in the United States rose from 1.99 to $2.18 \%$ of all hip arthroplasties and 2.05 to 
$2.18 \%$ of all knee arthroplasties between 2001 to 2009 [2]. The economic impact of the management of these infections is substantial, with a projected net cost of $\$ 1.62$ billion in 2020 [2].

In contrast to the comprehensive recommendations available for PJI treatment [3], other complications that can arise following a prosthetic joint implantation are not as well understood. One such complication is the incidence of calcium pyrophosphate deposition disease (CPPD), also known as pseudogout, typically characterized by sudden stiffness, pain and swelling at a native joint site. Prevalence estimates for this disease range from $4-7 \%$ of adults in the U.S and Europe, with the knee being the most common site affected $[4,5,6]$. In terms of pathophysiology in native joints, the first major step in this disease process is the formation of calcium pyrophosphate crystals in the pericellular matrix of cartilage, aided by the production of large amounts of extracellular inorganic pyrophosphate by local chondrocytes [7]. These crystals are responsible for the pain that patients experience at the joint site, as they can stimulate inflammatory changes via activation of the NLPR3 inflammasome and can accelerate the production of production of locally destructive matrix metalloproteinases and prostaglandins [8,9]. Unfortunately, the pathophysiology of pseudogout at prosthetic joint sites has not been clearly delineated, though it has been suggested that crystal formation and deposition can occur within synovial membrane remains or neosynovium that develops around the prosthetic joint [10].

Individual case reports have suggested that the initial presenting symptoms of pseudogout in an implanted joint are strikingly similar to those of an acute joint infection, with common complaints of tenderness, pain, swelling, and fever [11,12]. Given that the management of these conditions differs significantly, it is important that clinicians have a comprehensive awareness of the specific clinical features, risk factors, and prognosis of both conditions to facilitate earlier diagnosis and management decisions. The goal of our study is to describe the demographics, clinical presentation, management, and long-term outcomes of patients with prosthetic joints and pseudogout seen at our tertiary care medical center, using a retrospective cohort study design. Our findings will expand upon the limited data that exists on this rare complication at prosthetic joint implant sites.

\section{Methods}

Our study was a retrospective case series conducted at our tertiary care institution utilizing electronic health records to identify relevant patients and abstract all data. All adult patients ( $>18$ years of age) with a history of a prosthetic joint implantation and post-implant pseudogout at the same joint site, who were evaluated at Mayo Clinic, Rochester, MN between January 1, 2000 and June 30, 2016, were identified through our electronic health record (EHR) system. Specifically, we performed a comprehensive EHR search query for eligible patients using a data abstraction tool called Data Discovery and Query Builder (DDQB), with multiple possible terminology combinations of CPPD and prosthetic joint, prosthetic joint sites, and prosthetic joint infection ("CPPD and prosthetic joint," "CPPD and THA," CPPD and TKA," "CPPD and PJI"). This gave us a total of 247 potentially eligible patients. Each of these patient records was then examined individually to select patients who had a prosthetic joint implant and post-implant pseudogout at the same site. Implant associated pseudogout was defined as the identification of calcium pyrophosphate dihydrate crystals in synovial fluid aspirates taken from the joint or periprosthetic fluid. This yielded a total of 26 patients. Exclusion criteria included age below 18 and any missing management and/or lab findings in the patient's EHR. After applying our exclusion criteria, 22 patients were finally included in the study. Authorization for use of clinical data for research purposes was previously attained from all patients at initial point-of-care, and Institutional Review Board (IRB) approval for the current study was obtained. Patients were categorized into two groups based on the presence of concomitant infection along with their positive crystal findings. Evidence of concomitant infection was defined as presence of positive synovial fluid cultures. All descriptive data for included patients was abstracted from the EHR onto a secure Excel spreadsheet. Data pertaining to demographics, clinical presentation, management, and outcomes were then analysed using IBM SPSS Statistics Version 22. An additional member of the study team reviewed the data spreadsheet consisting of collected variables and final statistics.

\section{Results}

There were 22 patients ( 15 males, 7 females) who had a prosthetic joint implantation with subsequent identification of CPPD crystals in joint fluid during the study period. Majority of patients $(95.5 \%)$ were Caucasian. The mean age for this cohort was 71 years (Range: 51-84). A total of 9 patients (5 males, 4 females) had concomitant negative joint fluid cultures. A total of 13 patients (10 males, 3 females) had concomitant positive joint fluid cultures. Specific organisms identified on these cultures varied greatly. 
The specific distribution of organisms identified was as follows: Staphylococcus aureus (3/13, 23\%), Streptococcus species $(5 / 13,38 \%)$, coagulase-negative staphylococci $(2 / 13,15 \%)$, and miscellaneous species (Peptoniphilus, Propionibacterium, mix of Exiguobacterium/Corynebacterium/Mycobacterium chelonae) $(3 / 13,23 \%)$.

Table 1 summarizes the major demographic characteristics for the overall cohort and for each individual group, in addition to providing a summary of the major findings regarding patient medical background. The most common site of implant was total knee arthroplasty, which accounted for 20 (90.9\%) of all cases of post-implant pseudogout. The most common indication for prosthetic joint placement was degenerative joint disease (DJD), which accounted for $90.9 \%$ of the replacement procedures in this cohort. The number of implants during a patient's lifetime was consistent between both groups, with an average of 2 procedures. With regards to social habits, among those with negative synovial fluid cultures, $66.7 \%$ reported current alcohol use, while $44.4 \%$ reported previous tobacco use. Comparatively, $31 \%$ of patients with concomitant infection reported current alcohol use and $46 \%$ reported previous tobacco use. Between both groups, there was only one patient who reported current tobacco use. Two of nine patients without concomitant infection (22.2\%) had a history of diabetes mellitus, compared to six of thirteen patients with concomitant infection (46.2\%). None of the patients in the overall cohort were found to have a medical history of hemochromatosis, hypophosphatemia, or hypomagnesemia. One patient, who belonged to the group with concomitant infection, had a history of hyperparathyroidism. Only four patients were found to have a history of previous gout or pseudogout, three of which belonged to the group with no evidence of concomitant joint infection. Of these cases of previous gout or pseudogout, three cases occurred at sites different from the currently affected prosthesis (wrist, right first metatarsophalangeal joint, left second toe), while one case occurred at the same site (affected knee).

Table 2 expands on the clinical presentation of both groups. Clinical features for patients without concomitant infection included pain $(100 \%)$, swelling at the joint (88.9\%), redness (33.3\%), fever $(22.2 \%)$, and decreased range of motion (ROM) $(100 \%)$. Findings were similar for patients with evidence of infection on synovial culture, with majority of patients reporting pain, swelling, and decreased range of motion. Notably, none of the patients without concomitant infection were found to exhibit drainage from the prosthesis site. The median time in between onset of symptoms and CPPD documentation was 7 days in patients without concomitant infection, compared to 1 day for patients with concomitant infection. In terms of the gap of time between time of prosthesis placement (or last revision) and onset of symptoms at the prosthesis site, $7 / 9$ (78\%) of patients without concomitant infection and 10/13 (77\%) of patients with concomitant infection began experiencing symptoms $>12$ months after their prosthesis was placed or last revised.

Table 1. Demographics and Medical Background of Patients with Prosthetic Joint and CPPD Documentation

\begin{tabular}{llll}
\hline Variable Name & Entire Cohort (n= 22) & Patients with concomitant joint infection (n=13) & Patients without concomitant joint infection (n=9) \\
\hline Age at diagnosis (years) & $71 \pm 9$ & $72 \pm 8$ & $71 \pm 11$ \\
Gender: & & & \\
- Male & $15(68.2)$ & $10(76.9)$ & $5(55.6)$ \\
- Female & $7(31.8)$ & $3(23.1)$ & $4(44.4)$ \\
Weight $(\mathbf{k g})$ & $92 \pm 21$ & $87 \pm 18$ & $97 \pm 25$ \\
Height $(\mathbf{c m})$ & $168 \pm 9$ & $170 \pm 9$ & $167 \pm 11$ \\
Ethnicity & & & \\
- Caucasian & $21(95.5)$ & $13(100)$ & $8(88.9)$ \\
- Hispanic & $1(4.5)$ & $0(0)$ & $1(11.1)$ \\
Site of affected implant & & & \\
- Left knee & $7(31.8)$ & $5(38.5)$ & $2(22.2)$ \\
- Right knee & $13(59.1)$ & $6(46.2)$ & $7(77.8)$ \\
- Left ankle & $1(4.5)$ & $1(7.7)$ & $0(0)$ \\
- Right elbow & $1(4.5)$ & $1(7.7)$ & $0(0)$ \\
Indication for original prosthesis & & & \\
- DJD & $20(90.9)$ & $12(92.3)$ & $8(88.9)$ \\
- Rheumatoid arthritis & $1(4.5)$ & $1(7.7)$ & $0(0)$ \\
- Septic joint & $1(4.5)$ & $0(0)$ & $1(11.1)$ \\
Total number of implants & $2[1-4]$ & $2[1-4]$ & $2[1-3]$ \\
Current alcohol use & $10(45.5)$ & $4(30.8)$ & $6(66.7)$ \\
Current tobacco use & $1(4.5)$ & $1(7.7)$ & $0(0)$ \\
Previous tobacco use & $10(45.5)$ & $6(46.2)$ & $4(44.4)$ \\
History of malignancy & $7(31.8)$ & $3(23.1)$ & $4(44.4)$ \\
History of heart failure/dysfunction & $9(40.9)$ & $5(38.5)$ & $4(44.4)$ \\
History of chronic renal disease & $5(22.7)$ & $3(23.1)$ & $2(22.2)$ \\
\hline
\end{tabular}




\begin{tabular}{llll}
\hline Variable Name & Entire Cohort $(\mathbf{n}=\mathbf{2 2})$ & Patients with concomitant joint infection $(\mathbf{n}=\mathbf{1 3})$ & Patients without concomitant joint infection $(\mathbf{n}=\mathbf{9})$ \\
\hline History of previous gout or pseudogout & $4(18.2)$ & $1(7.7)$ & $3(33.3)$ \\
Antibiotics received prior to time of & $10(45.5)$ & $6(46.2)$ & $4(44.4)$
\end{tabular}

joint aspiration

Values are presented as mean $\pm \mathrm{SD}$, median [min-max] or $\mathrm{n}(\%)$.

Table 2. Clinical Presentation of Patients with Prosthetic Joint and CPPD Documentation

\begin{tabular}{|c|c|c|c|}
\hline Variable Name & $\begin{array}{l}\text { Entire } \\
\text { Cohort } \\
(n=22)\end{array}$ & $\begin{array}{l}\text { Patients with } \\
\text { concomitant } \\
\text { positive synovial } \\
\text { fluid cultures }(n=13)\end{array}$ & $\begin{array}{l}\text { Patients with } \\
\text { concomitant negative } \\
\text { synovial fluid cultures } \\
(n=9)\end{array}$ \\
\hline Pain & $21(95.5)$ & $12(92.3)$ & $9(100)$ \\
\hline $\begin{array}{l}\text { Pus or visible } \\
\text { drainage }\end{array}$ & 3 (13.6) & $3(23.1)$ & $0(0)$ \\
\hline Swelling & 17 (77.3) & $9(69.2)$ & $8(88.9)$ \\
\hline Redness & $8(36.4)$ & $5(38.5)$ & $3(33.3)$ \\
\hline Tachycardia & $2(9.1)$ & $2(15.4)$ & $0(0)$ \\
\hline Fever & $6(27.3)$ & $4(30.8)$ & $2(22.2)$ \\
\hline $\begin{array}{l}\text { Decreased range of } \\
\text { motion }\end{array}$ & $20(90.9)$ & $11(84.6)$ & $9(100)$ \\
\hline
\end{tabular}

Values are presented as $\mathrm{n}(\%)$.

The majority of patients $(85.7 \%)$ underwent initial imaging of the affected prosthesis, including all patients without concomitant infection and $75 \%$ of patents with concomitant infection. The most common modality was plain film radiography. Plain films for all patients with negative synovial fluid cultures revealed a normal fixed implant without periprosthetic lucency, with only one patient having notably significant bone erosion. On the other hand, patients with concomitant infection had more abnormalities, with some patients presenting with loose bodies and fluid collection with gas locules.

Blood cultures were negative for all patients. In patients without concomitant infection, the mean values of systemic CRP and ESR were $83 \mathrm{mg} / \mathrm{L}$ and 71 $\mathrm{mm} / \mathrm{hr}$, respectively. In patients with concomitant infection the mean CRP and ESR were $180 \mathrm{mg} / \mathrm{L}$ and $50 \mathrm{~mm} / \mathrm{hr}$ respectively. The leukocyte count and neutrophil count for both groups were elevated above normal. In patients without concomitant infection, the mean joint fluid cell count was 19892 cell/ $\mu \mathrm{L}$ and polymorphonuclear neutrophil (PMN) \% was 80. In patients with concomitant infection, the mean cell count was 70226 cell/ $\mu \mathrm{L}$ and PMN \% was 93. More than half of all patients in the overall cohort had synovial fluid that appeared bloody.

$45.5 \%$ of patients in the overall cohort received or had been on antibiotics prior to the time of joint aspiration $(44.4 \%$ of patients with negative synovial fluid cultures, $46.2 \%$ of patients with positive synovial fluid cultures). Median gap of time between antibiotic start date and joint aspiration for patients who had received pre-aspiration antibiotics was 1 day for both groups. Four of nine patients without concomitant infection and twelve of thirteen patients with concomitant infection were managed with a surgical procedure, most commonly irrigation and debridement. Only four patients in the overall cohort received targeted anti-inflammatory therapy post CPPD documentation, and all four belonged to the group without evidence of infection. Table 3 further delineates key management steps and laboratory findings for both groups.

Table 3. Management of Patients with Prosthetic Joint and CPPD Documentation

\begin{tabular}{|c|c|c|c|}
\hline Variable Name & $\begin{array}{l}\text { Entire Cohort }(n= \\
22)\end{array}$ & $\begin{array}{l}\text { Patients with concomitant positive synovial } \\
\text { fluid cultures }(n=13)\end{array}$ & $\begin{array}{l}\text { Patients with concomitant negative synovial fluid } \\
\text { cultures }(n=9)\end{array}$ \\
\hline $\begin{array}{l}\text { Time from symptom onset to CPPD } \\
\text { finding (days) }\end{array}$ & $2.5[0-96]$ & $1[0-11]$ & $7[1-96]$ \\
\hline Initial blood culture completed & $16(72.7)$ & $10(76.9)$ & $6(66.7)$ \\
\hline - Positive result & $0(0)$ & $0(0)$ & $0(0)$ \\
\hline Initial imaging obtained & $21(95.5)$ & $12(92.3)$ & $9(100)$ \\
\hline $\mathrm{CRP}(\mathrm{mg} / \mathrm{L})$ & $141 \pm 99$ & $180 \pm 103$ & $83 \pm 58$ \\
\hline ESR $(\mathrm{mm} / \mathrm{hr})$ & $58 \pm 32$ & $50 \pm 24$ & $71 \pm 40$ \\
\hline $\begin{array}{l}\text { Leukocyte count } \\
\left(\times 10^{\wedge} 3 / \mu \mathrm{L}\right)\end{array}$ & $10 \pm 4$ & $11 \pm 4$ & $9 \pm 3$ \\
\hline $\begin{array}{l}\text { Neutrophil count } \\
\left(\times 10^{\wedge} 3 / \mu \mathrm{L}\right)\end{array}$ & $8 \pm 3$ & $8 \pm 4$ & $7 \pm 2$ \\
\hline \multicolumn{4}{|l|}{ Primary synovial fluid appearance } \\
\hline - Cloudy & $8(36.4)$ & $5(38.4)$ & $3(33.3)$ \\
\hline - Bloody & $12(54.5)$ & $6(46.2)$ & $6(66.7)$ \\
\hline - Purulent & $2(9.1)$ & $2(15.4)$ & $0(0)$ \\
\hline $\begin{array}{l}\text { Synovial fluid total nucleated cells (cell/ } \\
\mu \mathrm{L} \text { ) }\end{array}$ & $49635 \pm 67098$ & $70226 \pm 80149$ & $19892 \pm 22318$ \\
\hline Synovial fluid PMN\% & $88 \pm 16$ & $93 \pm 6$ & $80 \pm 23$ \\
\hline Invasive procedure at prosthetic site & $16(72.7)$ & $12(92.3)$ & $4(44.4)$ \\
\hline - Irrigation and debridement & $12(75)$ & $9(75)$ & $3(75)$ \\
\hline - Resection arthroplasty & $3(18.75)$ & $3(25)$ & $0(0)$ \\
\hline - Synovial biopsy alone & $1(6.25)$ & $0(0)$ & $1(25)$ \\
\hline $\begin{array}{l}\text { Targeted anti-inflammatory drug } \\
\text { received post-CPPD crystal finding }\end{array}$ & $4(18.2)$ & $0(0)$ & $4(44.4)$ \\
\hline
\end{tabular}

Values are presented as mean $\pm \mathrm{SD}$, median [min-max] or $\mathrm{n}(\%)$. 
Table 4. Outcomes of Patients with Prosthetic Joint and CPPD Documentation

\begin{tabular}{|c|c|c|c|}
\hline Variable Name & $\begin{array}{l}\text { Entire Cohort }(n= \\
\text { 22) }\end{array}$ & $\begin{array}{l}\text { Patients with concomitant positive synovial fluid } \\
\text { cultures }(n=13)\end{array}$ & $\begin{array}{l}\text { Patients with concomitant negative synovial fluid } \\
\text { cultures }(n=9)\end{array}$ \\
\hline $\begin{array}{l}\text { Time to reported resolution of symptoms } \\
\text { (days) }\end{array}$ & $8[1-1464]$ & $8[1-1464]$ & $9.5[4-63]$ \\
\hline $\begin{array}{l}\text { Relapse and/or continuation of symptoms at } \\
\text { same site }\end{array}$ & $10(45.5)$ & $6(46.2)$ & $4(44.4)$ \\
\hline Future surgical intervention at same site & $7(31.8)$ & $6(46.2)$ & $1(11.1)$ \\
\hline \multicolumn{4}{|l|}{ Most recent status } \\
\hline - Lost to follow-up & $3(13.6)$ & $1(7.7)$ & $2(22.2)$ \\
\hline - Alive & $12(54.5)$ & $8(61.5)$ & $4(44.4)$ \\
\hline - Death & $7(31.8)$ & $4(30.8)$ & $3(33.3)$ \\
\hline Cause of Death & Total: 7 & Total: 4 & Total: 3 \\
\hline - Joint Infection-related & $0(0)$ & $0(0)$ & $0(0)$ \\
\hline - Joint Infection-unrelated & $6(85.7)$ & $3(75)$ & $3(100)$ \\
\hline - Unknown & $1(14.3)$ & $1(25)$ & $0(0)$ \\
\hline
\end{tabular}

Values are presented as mean $\pm \mathrm{SD}$, median [min-max] or $\mathrm{n}(\%)$.

The median time between onset and resolution of symptoms was 9.5 days among patients without evidence of concomitant infection and 8 days among patients with evidence of concomitant infection. A total of 10 patients in the overall cohort (45.5\%) had either relapse or continuation of symptoms after initial treatment. Relapse diagnoses among patients who had negative synovial fluid cultures included new PJI (1 case) and pain/functional deficit (3 cases). Relapse diagnoses among patients who had concomitant infection include repeat/continued PJI (3 cases) and 1 case of each of the following: growth of mycobacterium in original synovial fluid culture (requiring resection arthroplasty), planned multi-part surgery (irrigation and debridement with spacer exchange after initial resection arthroplasty), and surgical site infection with drainage and sinus tract formation. Of the 7 patients who required future surgical intervention at the affected joint, 6 had had initial evidence of concomitant infection. Finally, of the 7 patients who expired at follow-up, 6 were attributed to joint infection-unrelated causes (Table 4).

\section{Discussion}

Although pseudogout has been fairly well studied in native joints, its prevalence and presentation in prosthetic joints has been underreported. The purpose of the current study was to expand upon the limited information in the literature on post-implant pseudogout, in terms of patient demographics, clinical presentation, management, and outcomes. We found that majority of our patients with post-implant pseudogout were Caucasian males, with the most commonly affected joint replacement being TKA for DJD. Clinical presentation of this cohort was qualitatively similar to the symptoms of a PJI, and the only tool that could distinguish the two from a clinical management standpoint was synovial fluid cultures and crystal analysis. Still, nearly half of patients in the overall cohort received antibiotics prior to joint aspiration and majority received additional surgical management, most commonly irrigation and debridement. Ten patients in the overall cohort $(45.5 \%)$ had either recurrence or continuation of symptoms after initial treatment. It must be noted that early antibiotic therapy prior to definitive diagnosis serves as the major limitation of the present study--- it could be argued that at least some of the patients within the group without concomitant infection actually did have a prosthetic joint infection that was masked by this early antibiotic use, with the calcium pyrophosphate crystals being an innocent bystander.

This study has some other limitations inherent to its retrospective and descriptive nature. Specific inferences cannot be drawn regarding the population of prosthetic joint pseudogout patients in general. In addition, the small sample sizes in each of the individual groups serve as another limitation that may exaggerate the face value of some of the reported percentages. To counter this, both absolute frequencies and percentages have been reported for relevant variables. Ultimately, the purpose of this paper was to present demographics, clinical presentation, management, and outcomes of this underreported cohort of patients in a descriptive format, to serve as a basis for future studies studying this unique population within different research paradigms.

Of note, it is difficult to provide a unified comparison of some of our demographic data with that of other studies, largely because the existing literature includes mostly individual case reports or series with low patient counts. Khalfaoui and Yassa summarized 13 published cases of pseudogout in prosthetic joints across multiple centers. Although they did not aggregate information regarding gender, mean age for their patients with post-implant pseudogout was 72.1 years [12]. Yahia et al. provided a descriptive overview of 7 cases of crystal-induced arthritis (any type) after arthroplasty with an age range in years of 67 to 79 [10]. Additional cases have 
ranged in age from 59 to 85 years $[13,14,15,16,17,18]$. More so than gender, the average age of these cases appears to be similar to the average age of our cohort of patients $(71+/-9$ years). To our knowledge, our study was one of the first to report the frequency of various comorbid conditions and social history of a cohort of patients with post-implant pseudogout.

Among the post-implant pseudogout cases in the literature described above, the most common type of affected arthroplasty was TKA. This was consistent with our findings in the current study, and parallels the fact that the highest prevalence of native pseudogout is at knee joints. Interestingly, despite THA being common in the general population, there were no cases of pseudogout at prosthetic hips in our final cohort. It must be noted that one potential hip case was identified in our search process, but this patient had to be excluded due to missing management data. Nevertheless, it is interesting to note that monoarticular hip involvement is rare even for pseudogout at native hip joints [19].

The clinical signs and symptoms seen with initial presentation of our patients were also largely consistent with the reported cases in the literature. Specifically, these patients predominately present with pain, swelling, and restricted range of motion at the affected joint. However, there is some disparity in terms of discharge or pus at the affected site. None of our patients with negative synovial fluid cultures exhibited this sign. This is in contrast with some of the reported cases, where patients did have significant local discharge or pus [18]. There was also some variation in terms of the presence of systemic symptoms like fever or tachycardia, which were relatively minimal in our overall cohort but present in some reported cases in the literature $[12,15,18]$. Taken as a whole, it is evident that the signs and symptoms of post-implant pseudogout can easily be misattributed to those of a PJI due to considerable overlap, encouraging the practitioner to adopt a more aggressive management approach that is recommended for that complication [3]. Though the presence of certain signs and symptoms such as discharge, pus, fever, and tachycardia might encourage placement of infection at a higher level on a practitioner's differential diagnosis, post-implant pseudogout should not be ruled out even in those cases.

Perhaps the most prominent differences between our cohort of post-implant pseudogout patients and reported cases in the literature are centered on management. As described previously, Holt et al. reported a case of a 72 year-old male who was initially misdiagnosed with left knee PJI [15]. The patient had negative synovial fluid cultures and imaging of the affected joint was negative for loosening. These findings were similar to those of patients without concomitant infection described in our cohort. This patient underwent irrigation and debridement and a synovial biopsy that revealed additional crystals. However, in this patient, antibiotics were discontinued and diclofenac sodium was initiated, and the patient made a full recovery in seven days. It is interesting to note that only four out of nine of our patients without concomitant infection were deliberately started on an anti-inflammatory agent for their pseudogout symptoms. Khalfaoui and Yassa summarized 13 published cases of pseudogout in prosthetic joints [12]. Though the presenting symptoms, physical exam findings, negative imaging findings, and aspiration analyses were largely consistent with our findings, the vast majority of the 13 pseudogout patients included in the review were treated conservatively with anti-inflammatory agents. In fact, only $23 \%$ of pseudogout patients (with no concomitant infection) were treated with a surgical approach such as an open washout. In contrast, $44.4 \%$ of our patients with no concomitant infection received an invasive procedure such as irrigation and debridement or synovial tissue biopsy. Differences in management approaches between different institutions and clinical settings are thus evident, which points towards the need for future prospective trials comparing the short and long-term effectiveness of these approaches.

Management data from our study further reinforces the overlap with common findings of prosthetic joint infection. Results of complete blood counts and serum inflammatory markers, which are measured as part of standard management protocol for any patient with this type of presentation, are essentially indistinguishable between infection and pseudogout patients. Further complicating the clinical picture is the fact that infection can sometimes serve as a trigger for pseudogout, while the latter can also serve as a nidus for infection. Thus, an elevated leukocyte count, neutrophil count, CRP, and ESR are essentially non-specific findings and must be supported by more conclusive tests and analyses, including synovial fluid aspiration and analysis using microbial cultures and crystal examination. Unfortunately, many patients receive empiric antibiotic treatment prior to establishment of a diagnosis, as seen in nearly half of our patients with negative synovial fluid cultures. It should be emphasized that the administration of antibiotics should be avoided in hemodynamically stable patients until culture of periprosthetic tissue or synovial fluid is ascertained. Once cultures are obtained, the differential diagnosis for a patient 
presenting with signs of a prosthetic joint infection with negative synovial fluid cultures should include a crystalline arthritis such as pseudogout. Perhaps the most compelling argument for this inclusion is the difference in management for pseudogout compared to a prosthetic joint infection. Unlike the latter, the former does not definitively require surgical management, thereby providing an avenue to cut costs while optimizing efficiency of care and patient comfort. Future research should explore paired comparisons between post-implant patients with pure pseudogout and PJI, matched on the basis of variables such as age, gender, and implant site.

\section{Conclusions}

Our study expands on the limited data that exists on the rare complication of post-implant pseudogout, including key demographics, clinical presentation, management strategies, and outcomes of this cohort. The most common implant type was TKA, accounting for $20(90.9 \%)$ of all cases of pseudogout. The most common indication for prosthetic placement was degenerative joint disease. Our data suggests strong resemblance in presentation between post-implant pseudogout and more commonly encountered PJI, based on similar exam and lab findings (pain, swelling, redness, decreased ROM, elevated ESR/CRP/PMN\%). Conclusive testing including synovial fluid aspiration with crystal and culture analyses are necessary before antimicrobial therapy is given, in order to reliably distinguish pseudogout from infection at prosthetic joint sites. Unfortunately, many patients receive antibiotics prior to establishment of an etiologic diagnosis with joint aspiration, as seen in nearly half of our pseudogout patients with negative synovial fluid cultures.

\section{Acknowledgements}

Merit P. George and Elie F. Berbari contributed to project design, IRB submission, data collection, statistical analysis, and manuscript preparation. Floranne C. Ernste, Aaron Tande, Douglas Osmon, and Tad Mabry contributed to data review and manuscript preparation.

\section{Abbreviations}

TKA: total knee arthroplasty; THA: total hip arthroplasty; PJI: prosthetic joint infection; CPPD: calcium pyrophosphate deposition disease (pseudogout); ESR: erythrocyte sedimentation rate; CRP: C-reactive protein; WBC: white blood cell; EHR: electronic health record; IRB: institutional review board; DJD: degenerative joint disease; ROM: range of motion; $\mu \mathrm{L}$ : microliter; $\mathrm{mg} / \mathrm{L}$ : milligrams per liter; mm/hr: millimeters per hour; PMN\%: polymorphonuclear neutrophils \%; kg: kilogram; $\mathrm{cm}$ : centimeter; SD: standard deviation.

\section{Competing Interests}

The authors have declared that no competing interest exists.

\section{References}

1. Maradit-Kremers H, Larson DR, Crowson CS, et al. Prevalence of total hip and knee replacement in the United States. The Journal of Bone and Joint Surgery. 2015; 97: 1386-1397.

2. Tande AJ, \& Patel R. Prosthetic joint infection. Clinical Microbiology Reviews. 2014; $27: 302-345$.

3. Osmon DR, Berbari EF, Berendt AR, et al. Diagnosis and management of prosthetic joint infection: Clinical practice guidelines by the Infectious Diseases Society of America. Clinical Infectious Diseases. 2013; 56: e1-e25.

4. Neame RL, Carr AJ, Muir K, \& Doherty M. UK community prevalence of knee chondrocalcinosis: Evidence that correlation with osteoarthritis is through a shared association with osteophyte. Annals of the Rheumatic Diseases. 2003; 62: 513-519.

5. Felson DT, Naimark A, Anderson J, et al. The prevalence of knee osteoarthritis in the elderly: The Framingham Osteoarthritis Study. Arthritis \& Rheumatism: Official Journal of the American College of Rheumatology. 1987; 30: 914-918.

6. MacMullan P, \& McCarthy G. Treatment and management of pseudogout: Insights for the clinician. Therapeutic Advances in Musculoskeletal Disease. 2012; 4: 121-131.

7. Rosenthal AK, \& Ryan LM. Calcium pyrophosphate deposition disease. New England Journal of Medicine. 2016; 374: 2575-2584.

8. Abderrazak A, Syrovets T, Couchie D, et al. NLRP3 inflammasome: From a danger signal sensor to a regulatory node of oxidative stress and inflammatory diseases. Redox Biology. 2015; 4: 296-307.

9. Reuben PM, Wenger L, Cruz M, \& Cheung HS. Induction of matrix metalloproteinase- 8 in human fibroblasts by basic calcium phosphate and calcium pyrophosphate dihydrate crystals: Effect of phosphocitrate. Connective Tissue Research. 2001; 42: 1-12.

10. Yahia SA, Zeller V, Desplaces $\mathrm{N}$, et al. Crystal-induced arthritis after arthroplasty: 7 cases. Joint Bone Spine. 2016; 83: 559-562.

11. Khalfaoui MY. Crystal arthropathy of the prosthetic joint. J Ost Arth. 2016; 1:111.

12. Khalfaoui MY, \& Yassa R. Crystal arthropathy following knee arthroplasty: A review of the literature. International Journal of Orthopedics. 2015; 2: 411-417.

13. Harato K, \& Yoshida H. Pseudogout in the early postoperative period after total knee arthroplasty. The Journal of Arthroplasty. 2013; 28: 374.e9-374.e11.

14. Hirose $\mathrm{CB}$, \& Wright RW. Calcium pyrophosphate dihydrate deposition disease (pseudogout) after total knee arthroplasty. The Journal of Arthroplasty. 2007; 22: 273-276.

15. Holt G, Vass C, \& Kumar CS. Lessons of the week: Acute crystal arthritis mimicking infection after total knee arthroplasty. British Medical Journal. 2005; 331: 1322-1323.

16. Kobayashi H, Akizuki S, Takizawa T, et al. Three cases of pseudogout complicated with unicondylar knee arthroplasty. Archives of Orthopaedic and Trauma Surgery. 2002; 122: 469-471.

17. Levi GS, Sadr K, \& Scuderi GR. Bilateral pseudogout 8 years after bilateral total knee arthroplasty. Orthopedic Clinics of North America. 2012; 43: e59-e62.

18. Sonsale PD, \& Philipson MR. Pseudogout after total knee arthroplasty. The Journal of Arthroplasty. 2007; 22: 271-272.

19. Kocyigit F, Kuyucu E, \& Kocyigit A. Monoarticular hip involvement in pseudogout. Case Reports in Rheumatology. 2015; 2015: 1-3. 